\title{
Stress management training program for nursing students in Saudi Arabia
}

\author{
Olfat A. Salem ${ }^{1,2 \star}$, Anoud Kaabi ${ }^{3}$, Bodoor Al shehri ${ }^{4}$ and Reem Sufyani ${ }^{3}$ \\ ${ }^{1}$ Nursing Administration and Education Department, College of Nursing, King Saudi University, Kingdom of Saudi \\ Arabia. \\ ${ }^{2}$ Nursing Administration Department, Faculty of Nursing, Menofia University, Egypt. \\ ${ }^{3}$ Nursing Department, King Saud Medical City, Riyadh, Saudi Arabia. \\ ${ }^{4}$ Quality and Patient Department, General Directorate of Health Affairs Nursing Administration in Riyadh Region, Saudi \\ Arabia.
}

Accepted 3 September, 2018

\begin{abstract}
The primary aim of this investigation was to explore the effects of stress management programs on stress levels in nursing students studying in Saudi nursing college. Stress management training help the nurse to cope up with professional challenges, in retaining workforce and assist in solving the issue of shortage of registered nurses. This research project is a quasi-experimental study and sample size for this study was 16 students selected by using random sampling method. The researcher developed a questionnaire to measure the effectiveness of the stress management program regarding the students' stress levels. Results revealed nursing students who did not attend the interventional program reported an increase in the level of stress index level. Nurses who have attended the stress management sessions showed changes in their stress levels, although the minimization in stress was detected at a minute level. The study did not deliver a definitive outcome of the effectiveness of stress management program in improving coping abilities among nursing students. The outcome of this study though has not been clear, it still holds some valuable implications for future endeavors in stress management scenario.
\end{abstract}

Keywords: Stress, stress management, nursing students, education.

${ }^{*}$ Corresponding author. E-mail: osalem@ksu.edu.sa.

\section{INTRODUCTION}

According to Richard Lazarus, "stress is a two-way process; it involves the production of stressors by the environment, and the response of an individual subjected to these stressors. These stressful features can be entrenched in environment, culture, and also in a particular mindset. This constant state of tension imposes a constant burden on an individual's psychological, physical, and social health (Ratanasiripong et al., 2015). In addition, a person experiencing stress can commit mistakes and errors in professional life or at school. However, stress can also induce benefits to a person, for instance, it can make a person in engaging in measures which may promote his personal growth and development, and it can also encourage a person toward challenges and stimulation, and helps a person in handling less stressful situations later in life (Regehr et al., 2013). It is imperative that every individual has his own capacity and competency in managing stress. In professional lives, nurses are facing numerous stresses each day in their academic work, clinical postings, attending patients, learning and practice, and need support in efficiently completing their studies (Khater et al., 2014).

It is better to develop some strategies that may serve as an incentive in reducing stress among nurses so that they can focus on their duties in an efficient manner. For 
this, stress management training help the nurse to cope up with professional challenges helps in retaining nurses' workforce and thus assist in solving the issue of shortage of registered nurses in health care system (Ruiz-Aranda et al., 2014). There is no doubt that nursing professionals are well known for their extensive workload and critical nature of their work. According to a study by Reeve et al. (2013), the clinical environment that nurses experience is a major cause of stress as health care professionals experience one of the highest levels of stress among all professionals. In this regards, nursing students have a no different situation. Song and Lindquist (2015) identified that predictability of nursing stress is always predestined, however, nursing students despite being prepared to face stress in their upcoming career path, experience stress at a higher level. These stresses are relating to immense workload, uncertainty about career choice, educational stressors and also feeling of being incompetent going through in the clinical environments (Klainin-Yobas et al., 2014).

\section{Background of the problem}

There has been a significant body of literature on nursing students wherein they were provided with stress management skills; however, there is a lack in researches in nursing students in Saudi Arabia. The nursing force in Saudi Arabia is faced with high levels of retention and minimizing numbers of prospect nurses; one of the causes of such issues is the stressful experiences that nurses go through and, accordingly, the reputation of nursing being a stressful profession (Labrague et al., 2018). And since nurses make up a significant part of the medical profession, the present study aims to conduct an investigation in stress management among nurses in Saudi Arabia. This study further intends to determine the efficiency of stress management plan in nursing students in Saudi Arabia. This examination will help the researchers in identifying the importance of stress management initiatives in students studying nursing science. Blomberg et al. (2014) in their research article, instigated that stress in students is perceived as a phenomenon which needs exclusive investigation. In this respect, the article identifies stress not only has a negative effect on their academic performance but also impacts on their interaction with lectures and schoolwork. Al-Zayyat and Al-Gamal (2014) also highlighted in a Jordanian sample that nursing students experience immense stress during and after their admissions in nursing schools.

Eight studies, from a systematic review by Labraguea et al. (2018), reported nurses' stress levels which ranged from moderate to high stress levels. Of these studies seven were of moderate quality and one of high quality. The studies revealed that the majority of nursing students reported experiencing moderate to high levels of stress while the minority reported experiencing low levels of stress. Similarly, the authors found evidence showing moderate to high stress levels in Saudi student nurses that originated mainly from heavy workloads and taking care of patients examining stress perceptions and coping strategies among Saudi nursing students.

The stress before their admission is caused by demanding requisites to get admitted in nursing schools and also financial pressures have their weigh on them. Consequently, stress among nursing students after they are admitted in nursing schools and universities is due to intensive academic demands, hectic schedules, assignment submissions, research papers, examinations and a lot of other tests and viva voce (Hensel and Laux, 2014). Wolf et al. (2015) in their research project depicted that nurses students experience a high level of stress due to lack of skills in time management, financial pressures, and educational commitments. There are possibilities for other stressors associated with excessive homework, difficulty in understanding assignments, and inability in feeling comfortable in a classroom environment. Determining students' stress status that may affect physical and psychological health is essential for increasing nursing education quality. Also, educators should determine whether nursing students have effective coping skills and act accordingly (Polat and Demir, 2014; Klainin-Yobas et al., 2014).

Van der Riet et al. (2015) implicated that in order to cope up with everyday professional stress and anxiety, nursing students need to be equipped with ways by delivering coping strategies and management programs.

Song and Lindquist (2015) also pointed out that stress management skills help nurse in further their nursing career. The author also assessed the stress levels in Irish nursing students and depicted that stress among them was proven to be an essential factor needing urgent consideration. Therefore, it is imperative that stress management programs need to be introduced in nursing institutions included in their respective syllabus. In a study by Hamaideh et al. (2016), it was found that stress interventional program in Saudi Arabia have been successful in improving stress management skills and abilities in nursing students. The authors have mentioned that reduction in stress may lead to increased nurses' retention rates in health care and thus in ways may minimize morbidity and mortality among patients. Furthermore, these initiatives tend to improve emotional wellbeing in nurses and reduce stress levels in them (Patterson, 2016). It is thus imperative that stress management programs when introduced in nursing curriculum at King Saudi University is influenced by stakeholders, the fundamental entity is the government, academic institutions and health care leadership.

At the most, health care providers need to identify the importance of nursing practice and the immense difference they can make in the medical and nursing sector in Saudi Arabia. Since, retention rates in nurses is 
low and therefore, this shortage of nursing workforce negatively affects patients' wellbeing and overall quality of care. This deficiency in the number of nurses is basically due to the fact that nurses tend to work for a prolonged period of time without break or minimal breaks (Alzayyat and Al-Gamal, 2014).

As a consequence, these nurses feel exhausted owing to workload and are dissatisfied from their professional perspective, few are depressed and others are simply stressed out. In the current times, it is a common observation for nurses to leave the profession for reasons of stress in their field of practice. Although the pay scale of nurses is relatively better, the stressed nature of their work and inability to cope up with these stressful events is limiting them in providing their patients with the optimum quality of care. There are several institutions which have suitably proposed solutions to this issue, the most worth mentioning is the recommendations from federal and Honour Society of Nursing. One of the objectives of presenting solutions was to show the health care leadership how nurses are crucial for the development of health care industry. Another aim was to reshape the existing nursing profession by recruiting young people into the workforce by making nursing attractive to them. This will male young population to study nursing and new blood will be introduced in nursing care. Moreover, development of a practice atmosphere will also be crucial where care providers can freely interact with each other's and thus they can build healthy, productive and meaningful professional relationships.

Chernomas and Shapiro (2013) entailed that although the number of students enrolling in nursing institutions has significantly increased over the past few years, this increase is insufficient in balancing the number of nurses leaving the profession. This means that currently, nurses' students are working overtime due to reasons of shortages in the workforce. This phenomenon is a reason of great stress and depression in nursing students, therefore stress management programs are significant to this issue. Another research conducted by Labrague (2014) depicted that school-based stress management programs allow the student to handle stress from early days of their studentship in nursing institutions and a small number of these students experience burnout. Some of these pupils were trained to change their attitude in perceiving stress and consider it as an opportunity to excel in their professional lives. Since, literature lack in substantial body of evidence in addressing stress levels in nursing students, there is an urgent need to carry out this study among nursing students at King Saud University.

\section{Research aim}

The primary aim of this investigation is to explore the effects of stress management programs on stress levels in nursing students studying in Saudi nursing college.

\section{Research hypothesis}

Nursing students who attended the stress management program will demonstrate reduction in stress levels as compared to those who did not.

\section{METHODOLOGY}

This research project is a quasi-experimental study, intended to measure the effectiveness of program introduced to manage stress among nursing students. Following is the pre-test, intervention and post-test for the experimental group, whereas control group is only provided with pre-test and post-test.

\section{Study setting}

This study is conducted in a nursing college in the city of Riyadh, Kingdom of Saudi Arabia. This college of nursing was established in the year 1976 and later transformed in the department of nursing along with eight other departments of Applied Medical Science in the year 1979. Since it is crucial to realise that nursing is a speciality of medical science, the department of nursing was then translated into an independent college in the year 2004. This college of nursing provides a comprehensive education which is exclusively designed to benefit Saudi health needs approximately teaching 1000 students. The mission of the college of nursing is to extract philosophies from evidence based education and practices.

\section{Sample}

The sample size for this study is $16(n=16)$ selected by using random sampling method. The random sampling method is used in this study as every participant have an equal chance of getting selected. Random numbers were written on a paper and fingers ran on them with eyes closed and numbers were picked up until the desired sample size was selected. The study sample is 16 nursing students of which 8 in the experimental group and 8 in the control group.

\section{Data collection}

The data was collected in two phases;

Phase 1: Developing and applying for a stress management program in the form of a lecture with clinical components for a 2 hour session each week for 2 months.

Phase 2: First, a pre-test was applied for both the control and experimental groups. Then, the stress management program was applied to the experimental group. And lastly, a post-test was given to both the control and experimental groups.

\section{Ethical consideration}

Permission was obtained from the nursing administration of the college. In doing so, ethical code of conduct was strictly adhered to in this study and verbal consent was obtained from all participants. All the respondents were notified that their personal data would be kept confidential and anonymity would be maintained.

\section{Data analysis}

The data was entered, verified and coded on a personal computer 
with the help of Statistical Package for Social Science (SPSS). Descriptive and inferential statistical analyses, in the form of T-test and multivariate tests, was performed to draw the outcomes of the study.

\section{RESULTS}

Half of the sample was aged from 26 to 39 years. 31.2 percent were diploma holders, 43.7 percent were with bachelor degrees and 25 percent with masters' degrees. 75 percent single and 25 percent married. Out of total sample size, 75 percent did not have a child. Furthermore, 56.2 percent of the participants work in general hospitals, and 43.7 percent in primary care units. Moreover, 81.2 percent were in the level 5 of nursing college.

Table 1 shows that the mean value of stress index level value before the initiation of a program for the experimental group was 72.23; in which the mean of students not regularly following the intervention was 70.6. Additionally, average scores stress index level for students after the stress management program was 71.67 and students who did not attend the program was
73.21. It is clear that there has been a change in the level index for stress in participants before and after the program, however, the change detected is substantially small and our main objective was to know that our change is essentially significant or not and whether it is affected by time or because of the interventional program. It appear that there is a significant relationship between the level of stress in comparison to prior and after attending the interventional program

Table 2 shows the multivariate tests for variables time which measure the stress level index overtime and group intervention which fallowed the program or not. The Pillai's Trace test show that the $(F=0.016, P=0.902)$ for time which means that there is no significant difference in the stress level index measured before and after, so students who are measured before and after had no significant difference between before and after. In addition, the Pillai's Trace for the interaction between time and group attending the program is $(F=0.999 .1$, $\mathrm{P}=0.902$ ) which means that there is no statistical difference in the stress level index measured before and after across group intervention.

Table 1. T-test results in comparison to stress levels prior and post interventional exposure.

\begin{tabular}{cccccccc}
\hline \multicolumn{2}{c}{ Before the training (stress level) } & & \multicolumn{2}{l}{ After the training (stress level) } & \multirow{2}{*}{ T-test } & \multirow{2}{*}{ P. value } \\
\cline { 1 - 2 } \cline { 5 - 6 } Experimental group & Control group & & Experimental group & Control group & & \\
\hline 72.23 & 70.6 & 71.67 & 73.21 & -2.245 & 0.032 \\
\hline
\end{tabular}

Table 2. Multivariate tests.

\begin{tabular}{llccccc}
\hline Effect & & Value & F & Hypothesis df & Error df & Sig. \\
\hline \multirow{3}{*}{ Time } & Pillai's Trace & .001 & $.016^{\mathrm{b}}$ & 1.000 & 29.000 & .902 \\
& Wilks' Lambda & .999 & $.016^{\mathrm{b}}$ & 1.000 & 29.000 & .902 \\
& Hotelling's Trace & .001 & $.016^{\mathrm{b}}$ & 1.000 & 29.000 & .902 \\
\hline
\end{tabular}

\section{DISCUSSION}

This study intends to evaluate the differences in nursing students who underwent an interventional training program in comparison to those who did not undergo a training program.

It is imperative that stress management program is vital for maintaining stress and anxiety among nursing students in Saudi Arabia. This intervention makes sure that students are continuously focused on their actual personalities and finding new ways to cope up with their academic as well as professional stress. Reeve et al. (2013) highlighted that if nurses do not feel stressed out from their professional duties, they tend to perform well. Al-Zayyat and Al-Gamal (2014) in their study indicated that stress management is pivotal for students in order for them to prepare themselves for upcoming professional lives. According to the results of this study, nursing students who did not attend the interventional program reported an increase in the level of stress index level. Consequently, it was also deduced that this intervention has been timely and is of great significance owing to the results of this study.

In this study, it was also depicted that the nurses who have regularly attended the stress management sessions showed changes in their stress levels, although the minimization in stress was detected at a minute level. The results of the study revealed that nursing students experienced a reduction in their stress levels as stress was correlated with their education level. The nursing students who were single experienced higher stress level as compared to married ones. Furthermore, respondents 
who had a married showed a lower level of stress and those who were bachelors showed a higher level of irritability and nervousness. The participants having a child and working in private hospital settings showed the lesser level of stress. Therefore, it is implicated that the induction of stress management program among nursing students is likely to have effect in association to minimization in stress and anxiety index level.

\section{Study limitations}

This study has some limitations; for instance, participants may not have followed the instructions when they make use of stress management techniques in their classroom and professional settings as instructed. It is also perceived that nursing students who practiced their stress-reducing techniques outside classroom settings may be more likely to effectively manage stress and perform better in their studies and examinations. Another limitation can be stress management program has delivered only for a 2-hour session each week for 2 months which may not be sufficient time for intervention to improve nurses' retention in their courses and performing well. It is significant to note that researcher has been with the participants for the first time incorporating this stress-reducing intervention, thus it is possible that the effectiveness may improve in the future.

\section{Conclusion}

According to the statistical results, the following conclusion can be drawn;

Our study did not deliver a definitive and clear outcome of the effectiveness of stress management program in improving coping abilities among nursing students. There are multiple reasons for that: the sample size was small, the study duration was short, and a valid tool to measure students' stress levels was missing.

\section{IMPLICATIONS AND RECOMMENDATIONS}

The outcome of this study though has not been clear, it still holds some valuable implications for future endeavors in stress management scenario. The findings of this study further suggested that there is a need to provide counselling services to nursing students who are really struggling with their stress issues. Our results also imply that several meditation exercises need to be a part of stress training and departments need to perform a regular assessment to ascertain the stress demands among students. There is also a need for systemic evaluation of such programs so that effective outcomes are implemented and these evaluations can be conducted through an ongoing monitoring network or another formal evaluation study can also be beneficial.
Our results also implicated that stress management intervention should be of a longer duration of time so that it would be easier to assess the potential improvement in coping skills and gain in knowledge among students. There is a substantiative need to perform a meta-analysis on the existing literature of stress management techniques in classroom settings can be critiqued and appraised. In the future study, initial assessment of nursing student stress level at the pre-test time can be beneficial.

\section{REFERENCES}

Alzayyat A, Al-Gamal E, 2014. A review of the literature regarding stress among nursing students during their clinical education. Int Nurs Rev, 61(3): 406-415.

Al-Zayyat AS, Al-Gamal E, 2014. Perceived stress and coping strategies among Jordanian nursing students during clinical practice in psychiatric/mental health courses. Int J Mental Health Nurs, 23(4): 326-335.

Blomberg K, Bisholt B, KullénEngström A, Ohlsson U, Sundler Johansson A, Gustafsson M, 2014. Swedish nursing students' experience of stress during clinical practice in relation to clinical setting characteristics and the organisation of the clinical education. $J$ Clin Nurs, 23(15-16): 2264-2271.

Chernomas WM, Shapiro C, 2013. Stress, depression, and anxiety among undergraduate nursing students. Int J Nurs Educ Scholarship, 10(1): 255-266.

Hamaideh SH, Al-Omari $\mathrm{H}$, Al-Modallal H, 2016. Nursing students' perceived stress and coping behaviors in clinical training in Saudi Arabia. J Ment Health, 26(3): 197-203.

Hensel D, Laux M, 2014. Longitudinal study of stress, self-care, and professional identity among nursing students. Nurs Educ, 39(5): $227-$ 231.

Khater W, Akhu-Zaheya L, Shaban I, 2014. Sources of stress and coping behaviours in clinical practice among baccalaureate nursing students. Int J Humanities Soc Sci, 4(6): 194-202.

Klainin-Yobas $\mathrm{P}$, Keawkerd $\mathrm{O}$, Pumpuang $\mathrm{W}$, Thunyadee $\mathrm{C}$, Thanoi W, He HG, 2014. The mediating effects of coping on the stress and health relationships among nursing students: A structural equation modelling approach. J Adv Nurs, 70(6): 1287-1298.

Labrague LJ, 2014. Stress, stressors, and stress responses of student nurses in a government nursing school. Health Sci J, 7(4): 424-435.

Labraguea L, McEnroe-Petitteb D, Santosc J, Edetd O, 2018 Examining stress perceptions and coping strategies among Saudi nursing students: A systematic review. Nurs Educ Today, 65: 192200.

Patterson SL, 2016. The effect of emotional freedom technique on stress and anxiety in nursing students: A pilot study. Nurs Educ Today, 40: 104-110.

Ratanasiripong P, Park JF, Ratanasiripong N, Kathalae D, 2015. Stress and anxiety management in nursing students: Biofeedback and mindfulness meditation. J Nurs Educ, 54(9): 520-524.

Reeve KL, Shumaker CJ, Yearwood EL, Crowell NA, Riley JB, 2013. Perceived stress and social support in undergraduate nursing students' educational experiences. Nurs Educ Today, 33(4): 419-424.

Regehr C, Glancy D, Pitts A, 2013. Interventions to reduce stress in university students: A review and meta-analysis. J Affective Disorders, 148(1): 1-11.

Ruiz-Aranda D, Extremera N, Pineda-Galán C, 2014. Emotional intelligence, life satisfaction and subjective happiness in female student health professionals: the mediating effect of perceived stress. J Psychiat Ment Health Nurs, 21(2): 106-113.

Song $Y$, Lindquist $R, 2015$. Effects of mindfulness-based stress reduction on depression, anxiety, stress and mindfulness in Korean nursing students. Nurs Educ Today, 35(1): 86-90.

Van der Riet P, Rossiter R, Kirby D, Dluzewska T, Harmon C, 2015. Piloting a stress management and mindfulness program for 
undergraduate nursing students: Student feedback and lessons learned. Nurs Educ Today, 35(1): 44-49.

Wolf L, Stidham AW, Ross R. (2015). Predictors of stress and coping strategies of US accelerated vs. generic baccalaureate nursing students: An embedded mixed methods study. Nurs Educ Today, 35(1): 201-205.
Citation: Salem OA, Kaabi A, Al shehri B, Sufyani R, 2018. Stress management training program for nursing students in Saudi Arabia. Int ResJ Med Med Sci, 6(3): 85-90. 